\title{
Widerstrebende Modernität. Die Brief- und Arbeitsbeziehung zwischen Rainer Maria Rilke und seinem Verleger Anton Kippenberg
}

Wo sich Literatur innerhalb eines Gefüges von diskursiven Regeln und Institutionen betätigt, kann sie nur in indirekter Weise als dasjenige fungieren, was sie ihrem Wesen nach zu sein beansprucht, nämlich Mitteilung. Im Brief jedoch scheint hiervon eine Ausnahme möglich, folgt er doch den impulsgetragenen Mechanismen spontaner, quasi-mündlicher Kommunikation, die in einem ergebnisoffenen Verfahren an ein direkt adressiertes Gegenüber gerichtet ist. Wie und wem gegenüber teilt sich im Kunstwerk die Individualität seines Erzeugers mit? Es gibt hierauf eine gewissermaßen, romantische‘ Antwort, die unter Auspizien der Moderne nur mehr als sentimentalische gelesen werden kann. Dabei zeigt sich, dass auch und gerade die Konventionen der epistolaren Mitteilung einer Kundgabe spontaner, unverstellter Subjektivität durchaus entgegenstehen. Und so gerät selbst dieser Diskursbereich tief in die Ambivalenzen moderner Autorschaft hinein. Was dabei jeweils an reflektierten Entfremdungserfahrungen mit verhandelt wird, soll zum Einstieg eine künstlerische Szene der Selbstbegegnung aus dem Spätstadium des goethezeitlich etablierten Bildungsromans veranschaulichen.

Im vierten Band von Gottfried Kellers Künstlerroman Der grüne Heinrich gelangt der Romanheld in der 1880 erschienenen, von bedachtvoller Erzählregie durchwirkten zweiten Fassung des Werkes irgendwo im ländlichen Süddeutschland auf ein Grafenschloss, wo er die angenehme Bekanntschaft einer jungen Dame macht, Dorothea bzw. Dortchen, die sich während seiner Ankunft mit einer Mappe von künstlerischen Arbeiten beschäftigt hatte. Es handelt sich um eine Reihe von ausgebreiteten Talentproben, die der erschöpfte Held seinerseits nur flüchtig zur Kenntnis nimmt, weil ihn der Schlaf des weitgereisten Abenteurers übermannt.

Ich blickte mit einer Art einschläfernden Wohlgefallens nach dem Tische hin, sah und hörte mit halboffenen Augen und Ohren noch eine Weile, was sie taten und sprachen, ohne darauf zu merken, bis ich wirklich einschlief. Auf einem Stuhle neben sich hatte das Fräulein eine

Alexander Honold, Universität Basel

ว Open Access. (c) 2021 Alexander Honold, publiziert von De Gruyter (c))BY-NC-ND Dieses Werk ist lizenziert unter der Creative Commons Attribution-Non-Commercial-NoDerivatives 4.0 International Lizenz. https://doi.org/10.1515/9783110712568-015 
umfangreiche Mappe stehen, aus welcher sie größere und kleinere Blätter nahm, die auf Bogen starken Papieres zu heften sie beschäftigt war, daß die Blätter geschützt und mit einem breiten Rande versehen wurden. ${ }^{1}$

Unterstützt wird die junge Grafentochter bei ihrer konservatorischen Tätigkeit von einer Gehilfin, unter deren Blicken und Zwischenrufen die Landschaftsdarstellungen aus der Kunstmappe eine muntere wechselseitige Kommentierung erfahren, die der halbwegs in Schlummer gesunkene Protagonist und Icherzähler allerdings nur sporadisch wiederzugeben vermag.

Ich schlief über dem Mädchengezwitscher, das sich bis hieher ohne meine Teilnahme mir ins Gehör geschmeichelt, wirklich ein, erwachte aber einige Minuten später über einer ganz nah vor mir stattfindenden wohllautenden Ausrufung meines Namens. Die Gärtnerin hatte nämlich nach einem Weilchen, indem sie das aufgezogene Blatt weglegte, in einer Ecke desselben Namen und Jahreszahl zufällig bemerkt und gesagt: ,Was steht denn hier geschrieben?‘ [/] ,Was wird da stehen!' hatte Dorothea erwidert, ,der Name des Künstlers, der die Studien gemacht hat; denn das nennt man Studien, Landschaftsstudien! Heinrich Lee heißt er, alles in dieser Mappe ist von ihm!' Dann hatte sie sich plötzlich selbst unterbrochen, nach mir hergesehen und gerufen: ,Wie kann man nur so gedankenlos sein! Das sind ja meine Schweizerlandschaften, wie Papa sagt!' [/] Als ich jetzt die Augen aufschlug, stand sie dicht vor mir und hielt einen großen Bogen, zierlich an den obern Ecken gefaßt, vor der Brust, wie eine Kirchenstandarte, den schönen Mund noch geöffnet von dem Ausrufe: ,Herr Heinrich Lee!' [/] Ich aber war schon so schlaftrunken, daß ich die ersten Augenblicke nicht wußte, wo ich mich befand. Ich sah nur ein reizendes Wesen vor mir stehen, das mit freundlichen Augensternen über ein Bild herblickte. Voll traumhafter Neugierde beugte ich mich vor und starrte auf das Bild, bis mir erst die Waldlandschaft als bekannt erschien und ich mich dann auch meiner Jugendarbeit erinnerte. ${ }^{2}$

Dass die jungen Damen, während sie die Blätter mit Schweizer Landschaftsdarstellungen durchgehen, sich durch diese wundersame Entdeckung nun mit dem Künstler höchstpersönlich konfrontiert sehen, der diese Bilder in Jugendjahren einmal mit großem Ehrgeiz angefertigt hatte, ist innerhalb des Erzählgefüges alles andere als Zufall, auch wenn beide Seiten durch die wechselseitige Enthüllung nun in ein ebenso überraschendes wie charmantes Zusammentreffen hineingezogen werden. Es ist dies von Seiten des nicht mehr ganz so jungen, in seinem Laufbahnehrgeiz eigentlich schon resignierten Künstlers jener Augenblick höchster Erfüllung, wenn einem das eigene Werk so unerwartet und mit höchstem Liebreiz entgegentritt; ein Bild, von „freundlichen Augensternen“ überglänzt. Dass hier der Vater des Mädchens im Stillen tätig gewesen war, indem er durch systematische Aufkäufe nahezu sämtliche Jugendwerke des hoffnungslosen Künstlers aufgespürt

1 Keller 1996, 741-742.

2 Keller 1996, 743-744. 
und für seine Privatsammlung erworben hatte, weil ihn die verzweifelte Lage des jungen Mannes gerührt hatte, werden die Figuren und Leser des Romans erst eine beträchtliche Weile nach diesem wie traumwandlerischen Zusammentreffen erfahren.

Anders als in der viel ungestümeren Erstfassung des Romans, wo sein künstlerisches Scheitern den jungen Helden ins krachende und ausweglose Elend gestürzt hatte, kommt in der altersmilden Zweitfassung sowohl im persönlichen wie im beruflichen Entwicklungsgang des Protagonisten ein rettender Grundimpuls zur Geltung, der nach langen Jahren vergeblichster Anstrengungen die Glückssuche des grünen Heinrichs doch noch ins Positive zu wenden verspricht. Hierzu gehört auch die Versicherung, dass das Künstlerwerk der Jugendjahre nicht umsonst getan, die Sammlung seiner Produkte nicht verloren sei; hat sie doch durch die heilende Intervention des Grafen dort eine neue Heimstatt gefunden.

Gerade die traumwandlerische Sicherung der Werke durch einen verständnisvollen Mäzen aber untergräbt, indem sie sich ,supra-realistisch' der romantischen Register des Wunderbaren bedient, die für den Kunst- und Literaturbetrieb der Moderne konstitutiven Mechanismen eines marktförmigen Distributionswesens, in dem die Kunstwerke als Waren auf dem intermediären Raum zwischen Produzenten und Rezipienten gehandelt und taxiert werden. Denn erst durch das Kräftespiel jener Aushandlungen, die im Falle der bildenden Kunst zwischen Kunsthändlern, Galeristen, Sammlern, Kritikern und öffentlichem Kunstgeschmack stattfinden, wird über individuelle Eingriffe und Vorlieben hinaus so etwas wie ein intersubjektiver Kriterienkatalog des Anerkennenswerten ermittelt, ein Diskurs mit seinerseits wertsetzender Autorität. Auf dieses schwierige Parkett muss sich der grüne Heinrich, zögerlich aus seinem Tagtraum erwacht, nicht mehr begeben. Sein Augenblick des Erfolgs ist deshalb weder wiederholbar noch vorbildlich.

Eine Produktion für den anonymen Markt, auf welchem sich der Künstler durch die von ihm und seiner persönlichen Präsenz unabhängige Wirkung seiner Produkte einen Namen verschafft, kommt unter den Bedingungen klandestinen aristokratischen Mäzenatentums, wie sie Kellers Zweitfassung als einzig erfolgsträchtig imaginiert, dezidiert nicht zustande und wird letztlich auch gar nicht (mehr) angestrebt. Damit aber verfehlt die Kunstproduktion Heinrich Lees trotz der ihr Überleben (bzw. dasjenige des Künstlers) gesichert habenden Patronage den für die bürgerliche Kunst- und Kulturproduktion maßgeblichen Aspekt einer vom Produzenten abgekoppelten öffentlichen Zirkulationsebene, auf der die Kunstwerke ihrem Publikum in Warenform gegenübertreten und gerade deshalb nach einheitlichen, tauschwertorientierten ästhetischen Standards beurteilt, bewertet und in Umlauf gesetzt werden können. Haftet am Auftritt der Kunstwerke noch das Wirken persönlicher Patronage, so vollziehen sie die Transformation zur marktgängigen Warenexistenz entweder gar nicht oder nur halbherzig; ihnen wird nicht das 
Schicksal moderner Entfremdungserscheinungen zuteil, bei welchen die Namen der Künstler losgelöst von ihrer persönlichen Existenz und Verfügungsgewalt zu Markenzeichen und Wertindizes auf dem Markte mutieren.

Die Rolle intermediärer Akteure gilt nicht allein für Objekte der bildenden Kunst, sie ist auch im Literaturbetrieb zu beobachten, wo Verleger und Buchhändler die Kommunikation zwischen den Autoren und ihrem Publikum durch ein Geflecht medialer und kommerzieller Institutionen organisieren. Gottfried Kellers Schwierigkeiten mit seinen Verlegern, mit der Einhaltung zugesagter Abgabetermine und vereinbarter Lieferumfänge sind legendär - so als hätte er sich standhaft geweigert, die Mechanismen des Betriebes als solche überhaupt zur Kenntnis zu nehmen. Wenn Keller eine Frühform seiner späteren Seldwyla-Novellen zunächst unter dem Arbeitstitel Galathea zu entwickeln begonnen hatte, in Anspielung auf den Mythos der von dem Bildhauer Pygmalion unglücklich begehrten Frauenstatue, dann wird daraus deutlich, dass der Autor die ebenso aporetische wie leidenschaftliche Beziehung des Künstlers zu seinen Geschöpfen keineswegs durch das Dazwischentreten kommerzieller Akteure und Regularien zu entwirren gedachte, ganz im Gegenteil. Vielmehr reihte sich Keller damit ein in die Liste derjenigen künstlerischen Produzenten, die wie E. T. A. Hoffmanns Juwelier Cardillac an einem Syndrom der direkten Objektbeziehung zu ihren Pretiosen leiden und die eigenen Werke letztlich von den Distributionswegen eines entfremdeten Betriebes fernzuhalten bzw. aus diesen wieder zurückzuholen trachten.

\section{2}

Das Fortleben romantischer Phantasmen der schöpferischen Ursprünglichkeit und nichtentfremdeten Wirkungsweise von Kunst ist durch das gesamte neunzehnte Jahrhundert hindurch und bis weit in die klassische Moderne hinein wirksam geblieben. Dies lässt sich beispielhaft an einem Autor wie Rainer Maria Rilke verdeutlichen, in dessen ästhetischen und poetologischen Vorstellungen sich massive und wiederholte Vorbehalte gegenüber den Mechanismen eines institutionalisierten und damit entfremdeten Kulturbetriebes bemerkbar machen, obwohl er in seiner schriftstellerischen Produktivität zugleich von diesen Mechanismen profitieren konnte und sie sich für die Verbreitung und Pflege des eigenen Werkes effektvoll zunutze zu machen verstand.

Im Sinne einer ambivalenten ästhetischen Grunddisposition kann bei Rilke (und in ähnlicher Weise auch beim Geistesverwandten Hugo von Hofmannsthal) von widerstrebender Modernität bzw. von spezifisch modernen Formen des Widerstrebens gegen Entfremdungssymptome und Marktmechanismen gesprochen 
werden. Als Haltung zeigt sich diese widerstrebende Modernität insbesondere an der Diskrepanz zwischen den genieästhetischen Vorstellungen, die sich der Autor als Dichter im emphatischen Sinne von seiner gleichsam ,metaphysischen“ Berufung machte, und den durchaus zeitgemäß eingesetzten publizistischen Verbreitungskanälen und Wirkungsformen, die insbesondere seit Rilkes dezidierter Bindung an den Leipziger Verleger Anton Kippenberg und dessen kulturkonservatives Programm zur erfolgreichen Etablierung des Autors im Sinne eines literarischen ,Markenzeichens' führten.

Schauplatz und Austragungsort dieser disparaten, widerstreitenden ästhetischen Orientierungen ist primär die Briefproduktion, gewissermaßen das ,parergische‘ Werk. Im Aufstieg der Briefgattung zwischen Goethezeit und Moderne zu einem dominanten Ausdrucks- und Kommunikationsmedium spiegelt sich die gesteigerte Bedeutung bürgerlicher Verkehrsformen und die damit einhergehende Literarisierung sowohl alltäglicher wie geschäftlicher Lebensvollzüge. Die professionelle Schriftsteller-Existenz umfasst deshalb am Ende des neunzehnten Jahrhunderts neben der tradierten Gattungstrias von erzählender, lyrischer und dramatischer Darstellungsart zunehmend auch noch die vierte Gattung des epistolaren Schreibens als öffentliche oder zumindest halböffentliche Mitteilungsform. ${ }^{3}$ Dabei wird die Epistolographie nicht nur in sich selbst mit literarischer Dignität ausgestattet und sozusagen ,werkfähig' gemacht, sie erweist sich auch als werkbildend im Sinne des von Steffen Martus beschriebenen Phänomens der Werkpolitik, ${ }^{4}$ denn über die Transportwege brieflicher Kommunikation können die Wirkungsdimensionen des entstehenden Euvres sowohl stimuliert wie auch stabilisiert werden.

Im Falle Rilkes, bei dem die literarische Produktion auffälligen rhythmischen Verdichtungen und intermittierenden Phasen der Stagnation zu unterliegen scheint, kommt durch einige mittel- oder längerfristig unterhaltene Briefbeziehungen eine Form von diskursiver Kontinuität ins Spiel, so dass der brieflich mitlaufende Selbstkommentar auch über Wechselfälle seiner dichterischen Inspiration gewisse Aufschlüsse zu geben vermag. ${ }^{5}$ Zwar sind auch in Rilkes Briefkontakten berufliche und private Korrespondenzen in funktionaler Hinsicht zu unterscheiden, doch bewegt sich das Gros des Briefverkehrs in einem Überlagerungsbereich, der die Sozialaspekte von Freundschaft, Beratung, Testpublikum, Netzwerk und Geschäftsbeziehung verknüpft. Zu künstlerischen Gemeinschaftsarbeiten führen Rilkes Briefbeziehungen kaum; doch schaffen Übersetzungsprojekte,

3 Vellusig 2000; Strobel 2006; Bohnenkamp u. Wiethölter 2008.

4 Martus 2007.

5 Grundlegend zu Rilkes Brief-Poetik und Korrespondenzen Schuster 2014. Vgl. ferner Honold u. Wirtz 2019. 
Mehrsprachigkeit und gelebte europäische Internationalität (vgl. Rilkes intensive Beschäftigungen mit Russland, Frankreich, Italien, Südspanien, Skandinavien) vielfache interkulturelle Anreicherungen.

Seine großen Briefwechsel führte Rilke überwiegend mit Frauen, wobei die Korrespondenz mit Lou Andreas-Salomé das Paradigma langwelliger Schwankungskurven, ${ }^{6}$ derjenige mit Magda von Hattingberg das Prinzip eruptiver Verdichtung markiert, ${ }^{7}$ so dass beide Briefwechsel damit eine Grundspannung von Rilkes Arbeitsweise beschreiben. Diachron sind am Briefwerk fundamentale poetologische Achsenverschiebungen abzulesen. So bereitet die Wiederaufnahme des Briefkontakts mit Lou Andreas-Salomé im Sommer 1903 das diaristische Diskursmodell der Aufzeichnungen des Malte Laurids Brigge vor, die fünfwöchige epistolare amour fou im Briefverkehr mit Magda von Hattingberg wiederum modelliert 1914 die eruptiven Produktionsschübe von 1912 und 1922 nach bzw. vor; die Korrespondenzen mit dem Leipziger Verleger Anton Kippenberg und mit dem nach 1919 eingerichteten Schweizer Gönner- und Freundeskreis entfalten Rilkes Werkpolitik im beständigen Oszillieren zwischen kleinteiligen Textlieferungen und zyklischem Sammlungsbegehren, zwischen diasporischen und ökumenischen Distributionsstrategien.

Eine der durch epistolare Zeugnisse gut dokumentierten Übergangsphasen bildet die Zeit nach Fertigstellung des ersten umfangreicheren Prosawerks, der Aufzeichnungen des Malte Laurids Brigge. Das Erscheinungsjahr des Romans, 1910, bringt weit über die Arbeitszeit im engeren Sinne auch die Wirrungen mindestens einer halben Dekade an unstetem Künstlerleben zum Ausdruck, wo nicht gerade zu ihrem Abschluss. Für Rilke, der mit dem Brigge erstmals in größerem Umfang auf die Notate eigener Paris-Impressionen und ihrer brieflichen Ausformulierung (vor allem gegenüber Lou Andreas-Salomé) hatte zurückgreifen können, war diese ungewohnte Umformungsarbeit des diaristischen und epistolaren Prosamateri$\mathrm{als}^{8}$ mit großen ästhetischen Skrupeln einhergegangen, zu deren psychologischer Überwindung wiederum die nun überwiegend brieflich geführte Beziehung zu Lou Andreas-Salomé Entscheidendes beitrug. Je mehr sich aber das Brigge-Konvolut tatsächlich zu einer eigenständigen, literarisch-fiktionalen Existenz verfestigte, desto stärker wird die Rolle der existentiellen Stabilisierung nun von einem anderen Briefverkehr des Autors übernommen, nämlich durch die im Jahr 1906 aufgenommene und parallel zu dieser Arbeit intensivierte Korrespondenz mit dem Insel-Verleger

6 Vergleichbares gilt auch für die Briefwechsel mit den Damen Thurn und Taxis, Nádherný von Borutin, Wunderly-Volkart.

7 Ähnlich gelagert sind Rilkes Briefwechsel mit den Dichterinnen Mitterer und Zwetajewa.

8 Vgl. hierzu Kammer 2019, 33-52. 
Anton Kippenberg ${ }^{9}$ - ein Arbeitsverhältnis, das in Rilke die Weichen für eine neue Werkphase stellen wird.

Mit dem Vertragsabschluss für das 1906 erscheinende Stunden-Buch beginnt die zwei Jahrzehnte währende Verbindung zu Anton und Katharina Kippenberg. Mit seinem Leipziger Verleger wechselt Rilke knapp 700 Briefe; in ihren Anfangsjahren ist die Korrespondenz von der allmählichen Vertrauensbildung und wachsender persönlicher Nähe gekennzeichnet. So erwärmen sich bei Kippenberg die Grußformeln in Gradationen von „Sehr geehrter Herr“ über „Mein lieber und sehr verehrter Herr Rilke“ bis zu „liebster Freund“. ${ }^{10}$ Von Beginn an stabilisiert Rilke die Beziehung durch Zusicherung von Exklusivität und erklärt sein „aufrichtiges Bedürfnis, meine kommenden Bücher endlich einheitlich unterzubringen“.11 Geschäftlich eint beide das Interesse, die Rechte an Rilkes früheren Publikationen Schritt für Schritt ebenfalls auf den Insel-Verlag zu übertragen - ein angesichts von Rilkes zunehmender Erfolgsträchtigkeit recht mühseliges Unterfangen, das erst mit der Übernahme des Cornet als Eröffnungsband der Insel-Bücherei sechs Jahre später zum vorläufigen Abschluss gelangt. ${ }^{12}$

Von Capri aus, am 11. März 1908, hatte Rilke an Kippenberg geschrieben, dass er die „Beziehung“ zu dessen Verlag nun durch eine langfristige vertragliche Bindung zu bekräftigen hoffe, und dass sich für ihn dieser Schritt wiederum mit der poetologischen Konsequenz verbinde, das eigene Schaffen künftig, auch aus kommerziellen Erwägungen heraus, stärker als bislang auf die Niederschrift literarischer Prosa auszurichten.

Ich bin mir bewußt, daß ein lyrisches Werk, geschäftlich gesprochen, als eine schwankende Sicherstellung anzusehen ist, auf die hin jede vorausgreifende Vereinbarung etwas Waghalsiges bedeuten mag. Soweit man das absehen kann, ist aber meine lyrische Arbeit (so endgültig sie auch in einzelnen Verwirklichungen sich darstellt und immer wieder darstellen wird) ein persönliches Besitzergreifen, eine Bewältigung der Außenwelt, hinter der sich andere Aufgaben, Verdichtungen und Lösungen vorbereiten; denn wenn ich mir jetzt mit aller Sorgfalt eine intensive Arbeitszeit zu sichern wünsche, so denke ich nicht nur an den Abschluß des nächsten Gedicht-Buches, sondern, mit ebenso großer Hingabe, an den Ausbau meiner Prosa und darüber hinaus an eine gewisse dramatische Notwendigkeit, die vielleicht eines Tages aus der bis zum Äußersten angewachsenen künstlerischen Spannkraft entspringt. ${ }^{13}$

9 Rilke 1995.

10 Kippenberg an Rilke, 07. November 1906; 04. November 1908; 15. Februar 1912. BW Kippenberg, Bd. I, 59, 127, 325.

11 Rilke an Kippenberg, 10. November 1906; BW Kippenberg, Bd. I, 60.

12 Im Mai 1913 folgen noch die von Langen übernommenen Rechte an Rilkes dramatischem Frühwerk Das tägliche Leben; „damit sind alle Ihre Bücher nunmehr endgültig im Insel-Verlag vereinigt“ (Kippenberg an Rilke, 30. Mai 1913; BW Kippenberg, Bd. I, 403).

13 Rilke an Kippenberg, 11. März 1908; BW Kippenberg, Bd. I, 100-101. 
Rilke, dessen materielle Verhältnisse nach dem Auslaufen seiner Stipendien ins Prekäre abzurutschen drohten, hoffte auf eine existenzsichernde und langfristige finanzielle Unterstützung durch Kippenberg. Um dem Verleger eine solche Verpflichtung plausibel zu machen, zeigt sich der Autor, der bis dahin seine lyrische Produktion nur zögerlich zu größeren publizistischen Einheiten zusammengestellt hatte, zu erstaunlich weitreichenden Konzessionen und sogar zu stilistisch-literarischen Anpassungen bereit. Rilke macht sich anheischig, sein bis dato auf symbolistische Lyrik eingeschränktes literarisches Portfolio um vermeintlich marktgängigere Produktsparten zu erweitern. Seine erstaunliche Bereitschaft zur Übernahme der unternehmerischen Perspektive, wiewohl sie für den angedeuteten Wandel der Gattungspräferenz nicht einmal ausschlaggebend gewesen sein mag, stellt innerhalb des brieflichen Netzwerks eine bis dahin ungekannte Geste partnerschaftlichen Entgegenkommens dar. Auch wenn seitens des Verlegers eine solche Verlagerung des Produktionsschwerpunktes in die Prosa gar nicht unbedingt opportun scheinen mochte, bekundet die Ernsthaftigkeit, mit welcher sich Rilke um das Wohlwollen Kippenbergs bemüht, eine ästhetische Verbindlichkeit, die über seine bisherigen Verlagsbindungen weit hinausgeht und das Bild eines der alltäglichen Welt entrückten Dichters zu revidieren zwingt.

\section{3}

Während der Fertigstellung des Buchmanuskripts zum Malte Laurids Brigge - ein Vorgang, der sich durch Krankheitsschübe des Dichters im Frühjahr 1909 um viele Monate verzögerte ${ }^{14}$ - kommt es in der Korrespondenz mit Kippenberg zu einer Intensivierung der Brieffrequenz und zu einer merklichen Steigerung des epistolaren Vertrautheitsgrades. Während in anderen Briefwechseln das regelmäßige Hin und Her der postalischen Sendungen auch dazu diente, die persönliche Begegnung zu ersetzen oder gar zu vermeiden, läuft die Intensivierung der Verleger-Korrespondenz in ihrer immanenten Dramaturgie folgerichtig auf die Anbahnung eines direkten Zusammentreffens der literarischen Geschäftspartner zu.

Befördert wird diese Entwicklung dadurch, dass der Dichter im Herbst des Jahres 1909 mit einem prima vista eher nebensächlichen und umständlich formulierten Anliegen an seinen Verleger herantritt. Es geschieht dieses während

14 „Durch die Art, wie Sie schließlich nach dem Fortgang des Malte Laurids Brigge fragen, erleichtern Sie mir eine Mittheilung, die diesmal leider allen Ihren guten Mittheilungen nicht entspricht. Es ist ausgeschlossen, daß ich das Buch zum August abschließe.“ (Rilke an Kippenberg, 21. Mai 1909, BW Kippenberg, Bd. I, 161). 
des letzten Stadiums der Arbeit am Brigge-Manuskript, als dem wieder in Paris wohnenden Schriftsteller bewusst wird, wie sehr sich durch die Arbeit mit unterschiedlichen Textstufen die Gefahr zusätzlicher Fehler und Ungereimtheiten erhöht, und dies ausgerechnet in der ohnehin bereits unter Zeitdruck stehenden Abschlussphase der Niederschrift. Es sei „völlig unwahrscheinlich“, so eröffnet Rilke sein Anliegen, dass ihm der Verleger in der folgenden Angelegenheit „rathen“ könne, doch erlaube er sich aus vertrauensvoller Gewohnheit dennoch den Schritt, Kippenberg sein Problem vorzutragen.

Die Frage voraus: Wüßten Sie durch zufällige Verlagserfahrung hier jemanden, der gut und zuverlässig mit der Schreibmaschine deutsche Abschriften, theilweise Diktate, liefern könnte? [...] Die ganze Angelegenheit sieht sehr nebensächlich aus, aber ich will Ihnen mittheilen, weshalb sie mir wichtig und ernst ist. [/] Von meinem Prosabuch ist die Hälfte da; vielleicht etwas mehr. Nun steht der Text in kleinen Taschenbüchern und einem älteren größeren Manuscript und ist schlecht zu übersehen; nicht das allein: im vergangenen Winter in zunehmender Erschöpfung und Unpäßlichkeit mühsam weiterarbeitend, habe ich, ganz gegen meinen sonstigen Gebrauch, mich zu nachlässiger und undeutlicher Aufzeichnung mancher Partieen gehen lassen; so daß eine gleichmäßige durchgehende Abschrift auch dadurch schon nöthig wird. Sie fehlt mir immer mehr. Nun wäre es ja, gerade bei der theilweise schlechten Handschrift des Manuscriptvorraths, am natürlichsten, wenn ich mich selbst ans Abschreiben des Vorhandenen machte. Ich würde damit, seit ich hier bin, schon begonnen haben, aber meine Gesundheit ist immer noch halb, und ich fürchte, vielleicht mit Recht, in der mechanischen Kopistenbeschäftigung mich rasch und unfruchtbar zu ermüden. ${ }^{15}$

Es dürfte dem Schreibenden dieser Zeilen nicht ganz leichtgefallen sein, dem Verleger so weitreichende Einsichten in die inneren Produktionsabläufe und deren krisenhafte Bestandteile zu eröffnen. Wenn Rilke, der für gewöhnlich über eine elegante und mit gestochener Klarheit vorangehende Handschrift verfügte, in diesem Falle über die mangelhafte Qualität seiner eigenen Notate Klage führen musste, so kommt darin eine enorme psychomotorische Anspannung seiner Schreibhand und ihrer überforderten Bewegungskontrolle zum Ausdruck. Derartige Symptome wiederum scheinen der Preis eines empfindlichen sensorischen Systems gewesen zu sein, wie es schon im quasi-technischen Konzept der ,Aufzeichnungen“ selbst angedeutet ist. ${ }^{16}$ In den Brigge-Notaten geht die graphische Radikalität des Auf-

15 Rilke an Kippenberg, 20. Oktober 1909; BW Kippenberg, Bd. I, 177.

16 Der Begriff der Aufzeichnung ist nicht lediglich Bestandteil des Romantitels, sondern sollte in Rilkes Werkabsicht den durchgängigen Diskursmodus des Textes bezeichnen und als solcher im Text auch fortlaufend angezeigt sein, wie der Dichter gegenüber seinem Verleger insistiert: „Nichtwahr: es bleibt dabei und man wird nicht vergessen, über alle Seiten in unserem Buch die Aufschrift ,Die Aufzeichnungen des / Malte Laurids Brigge‘ zu setzen?“ (Rilke an Kippenberg, 16. April 1910; BW Kippenberg, Bd. I, 204). 
zeichnens gelegentlich so weit, dass eine literale Decodierbarkeit der Schriftspuren teilweise schon nicht mehr gegeben war. Die Spur der aufzeichnenden Hand musste also ins Reine geschrieben, musste zur Ergebnissicherung und Weitergabe im Publikationsprozess nochmals sorgsam transkribiert werden.

Die naheliegende Möglichkeit, dabei gleichsam ,sich selbst' nochmals aufzuzeichnen, dem eigenen Schreiben hinterher, scheidet Rilke sogleich aus Gründen der mechanischen Erschöpfungsgefahr aus. Ein zweiter, ungenannter Grund wiegt schwerer: Rilke muss möglichst deutlich und wirkungsvoll zwischen dem kreativen und dem reproduktiven Anteil im Akt des Schreibens unterscheiden, sonst droht jede Abschrift wieder neue Umschriften vorzunehmen, die dann nochmalige Transkription erfordern würden, und so fort. Für eine institutionalisierte Produktion von Literatur braucht es ein Minimum an medienästhetischer Arbeitsteilung, ein differenziertes Zusammenspiel von Sendefunktion und Übertragungsweg, bei welchem der Dichter tunlichst nicht alle Komponenten selbst übernehmen sollte.

Der Briefschreiber scheint bei dem vorgetragenen Anliegen nicht ernstlich darauf gehofft zu haben, es ließen sich solche deutschsprachigen Sekretariatsarbeiten auch in Paris bewerkstelligen; diese nur hypothetische Erwägung dient im Fortgang des Schreibens vielmehr zur Anbahnung einer unverhüllten Selbsteinladung, mit welcher der Autor in die unmittelbarste Nähe seines Verlegers zu gelangen und an dessen administrativen Ressourcen zu partizipieren sucht. „Es würde natürlich die Weiterarbeit ungemein erleichtern“, so fährt Rilke fort, „wenn ich das Bestehende klar und endgültig vorher sichern könnte. Ist aber keine Möglichkeit dazu (was mir fast ausgemacht erscheint) so will ich meine Arbeit zunächst fortsetzen, unter der Vornahme, ehe ich wieder weiter fortreise, im Winter also, für etwa acht Tage nach Leipzig zu kommen, wo Sie mir sicher einen genauen Abschreiber empfehlen und an die Hand geben könnten. In gewisser Weise wäre ja dieser Ausweg der solideste und auch mir liebste“, so bekräftigt der Autor den Gang seiner Überlegungen, die ihn geradezu zwingend zum Sitz seines Verlages als seinem naturgegebenen Unterstützungsort geführt haben. ${ }^{17}$

Anton Kippenberg beeilte sich, in einem sofortigen Antwortbrief die nur indirekt geäußerte, gleichwohl unziemlich aufdringliche Anfrage seines Autors mit einer herzlichen Einladung zu beantworten.

Mein sehr verehrter und lieber Herr Rilke! [/] Die Kalamität, in die Sie die Notwendigkeit, den Laurids Brigge auf dem Wege des Diktats umschreiben zu lassen, versetzt hat, ist mir, offen gesagt, sehr erwünscht gekommen. Denn so gibt sie mir die Aussicht, Sie in naher Zeit endlich selbst in Leipzig begrüßen zu dürfen. In Paris eine deutsche Sekretärin zu suchen, scheint mir ziemlich aussichtlos zu sein, und da Sie Bedenken tragen, das Manuskript von

17 Rilke an Kippenberg, 20. Oktober 1909; BW Kippenberg, Bd. I, 178. 
sich zu geben - in welchem Falle ich es hier natürlich auch mit aller erdenklichen Sorgfalt hätte abschreiben lassen - so scheint es auch mir als das weitaus zweckmässigste, dass Sie für 8 Tage oder länger nach Leipzig kommen und hier das Diktat vornehmen. Natürlich wohnen Sie bei uns, und es steht Ihnen ausser Ihrem Schlafzimmer ein Turmzimmer ganz zu Ihrer Verfügung, das sehr sonnig und hell ist und uns immer recht geeignet geschienen hat, um einmal einen Poeten aufzunehmen. Der Blick in grüne Bäume, den man von dort im Sommer hat, wird nun zwar im Winter fehlen. Wenn es eben sich ermöglichen lässt - und ich glaube das, da im Winter weniger zu tun ist - so würde ich meine Sekretärin bitten, die Arbeit zu übernehmen. ${ }^{18}$

Der Verleger besaß die Größe, die scheinbar nebensächliche Anfrage seines Autors und dessen Bitte um Unterstützung bei der Manuskripterstellung mit denkbar freundlichstem Entgegenkommen zu beantworten; er erfasste dessen Anliegen dabei gerade auch in seinen symbolischen Bezügen mit intuitiver Treffsicherheit. Denn in diesen beiden Briefen geht es, über die pragmatischen Aspekte der Textabschrift hinaus, im Kern um eine Art Modellszene der Verfertigung von Literatur überhaupt. Der Autor steht seinerseits zwar am Ausgangspunkt der Texthervorbringung, doch kann er alleine ein verwertbares Manuskript eben nicht liefern, sondern ist dazu auf fremde Hilfe, auf ein Zusammenspiel mit anderen Kräften angewiesen. Neben die Schreibtechnik der bei diesem Werk sogar titelgebenden „Aufzeichnungen“ tritt im Zuge der Manuskripteinrichtung eine duale Produktionsform von Diktat und Niederschrift, ummantelt schließlich durch eine mehr als nur geschäftsmäßige Arbeitsgemeinschaft des Schriftstellers mit seinem Verleger.

Auf das Angebot, die diktierende Abschrift aus dem Brigge-Manuskript als Hausgast seines Verlegers vorzunehmen, geht Rilke mit überschwänglicher Freude ein - der Partner hat seinen Autor mit dieser Einladung besser verstanden als dieser sich selbst. „Ihre Freundschaft, mein lieber und verehrter Herr Doktor, hat einen so glücklichen Griff, daß ich diese amorphe Sache, deren Oberfläche ich ganz zu kennen meinte, nur in die Hand nehmen mußte, um mir eine neue schöne Seite zuzukehren."19 Rilke bittet Kippenberg, den Gastaufenthalt zeitlich „so einzuordnen, wie er ihnen paßt“, und der Einladende versichert sogleich, Rilke möge doch seinen „Aufenthalt in Leipzig nicht gar zu knapp bemessen“, so dass sich ,uns auch ausserhalb der Diktatarbeiten Gelegenheit und Musse bieten möge, einander recht kennen zu lernen.“20

Es ist ein wechselseitiges Werben umeinander, das diese Verleger-Korrespondenz mit ihrem altmodischen Stil zugleich in wirtschaftlich so erfolgreiche Bahnen lenkt. In der erst mit Kippenberg für Rilke erreichbaren Freundschaftsbindung er-

18 Kippenberg an Rilke, 23. Oktober 1909; BW Kippenberg, 178-179.

19 Rilke an Kippenberg, 26. Oktober 1909; BW Kippenberg, Bd. I, 179; das folgende Zitat: 180.

20 Kippenberg an Rilke, 1. November 1909; BW Kippenberg, Bd. I, 180. 
langt die verlegerische Geschäftsbeziehung eine persönliche Intensität und Durchdringung, deren symbiotische Nähe hierbei zum gelebten Ereignis wird, sobald sich der Schriftsteller tatsächlich anschickt, im Hause seines Verlagschefs als eine Art writer in residence in das für den Poeten bereits vorbestimmte und vorbereitete Turmzimmer einzuziehen. Dabei ist Kippenberg emotional versiert genug, die symbolische Handlungsebene dieser Einladung als die ausschlaggebende zu unterstreichen, denn für das praktische Manuskriptproblem selbst hätten auch andere, unaufwändigere Lösungen bestanden.

Doch erst die leibhaftige Einquartierung des Dichters in das ihm bestimmte Turmzimmer schmiedet das Geschäftsverhältnis von Autor und Verleger zu einer synergetischen Nähe-Allianz um, bei der die beiden Hauptakteure der literarischen Produktion sich zu einem direkten physischen Konglomerat verbinden. Rilke meldet sich nach erfolgter Ankunft am 12. Januar 1910 in Leipzig bei seinem Verleger an, ${ }^{21}$ das Manuskript hat er unterdessen mit eingeschriebener Post bereits vorausgehen lassen. Vom 12. bis zum 31. Januar zieht der Dichter dann wie vereinbart in das für ihn bestimmte Turmzimmer von Kippenbergs Villa in der Richterstr. 2, wo er in wenigen Tagen die Diktatabschrift seines Manuskriptes erstellen lassen kann. Dadurch wird für einen kurzen, aber folgenreichen Zeitraum nun in concreto vollzogen, worauf im Grunde schon Kellers Grüner Heinrich mit seinem Tagtraum von den zurückgekauften Jugendwerken zugesteuert war: die Überwindung institutioneller Entfremdungserscheinungen im Produktions- wie Rezeptionsprozess des bürgerlichen Kunstbetriebes.

\section{4}

Konstitutiv für den literarischen Betrieb der Moderne ist ein striktes Regiment von Distanzen und wechselseitigen Ausschlussregeln: der Schriftsteller schreibt in Abwesenheit seines prospektiven Publikums, dieses hört bzw. liest anstelle des sich mitteilenden Verfassers selbst dessen intermediären Text; die dazwischentretenden Akteure wie Verlag, Buchhandel und Presse sorgen für ein geregeltes, differenziertes Zusammenspiel bei Wahrung der arbeitsteiligen Abstände und Zuständigkeiten. Rainer Maria Rilke ist in seinem Selbstverständnis als Autor niemals zur Gänze in diesem funktional differenzierten Diskurssystem angekommen; er schrieb im Sog eines vermeintlichen Auftrags persönlicher oder gesellschaftlicher Natur, gerichtet an ein lebensweltlich erfahrbares Publikumsmilieu und jeweils

21 „Ich spreche nicht selbst ins Telephon, schreibe deshalb lieber“ (12. Januar 1910; BW Kippenberg, Bd. I, 185). 
angespornt vom brieflichen Drängen des Verlegers oder anderer Kooperationspartner. Es kann und darf unter Bedingungen moderner Arbeitsteilung naturgemäß nicht die Normalität literarischen Produzierens sein, dass Schriftsteller bei ihren Verlegern im Hause mitwohnen; vielmehr handelt es sich hierbei um ein nur je als Ausnahme- und Grenzfall symbolischer Art denkbares Geschehen. Die eigentliche Wirkung des Vorgangs besteht denn nicht nur in der dabei tatsächlich erfolgten Manuskripterstellung, sie liegt vor allem in der diskursstabilisierenden Ausstrahlung, die von dem zeitlich begrenzten Ereignis für das fortlaufende Hin und Her des Briefverkehrs ausgeht.

Ganze acht Briefe, die im Voraus zwischen Autor und Verleger noch gewechselt wurden, nehmen auf Planung, Ablauf und Modalitäten des vorgesehenen Dichter-Aufenthaltes beim Leipziger Verleger Bezug. Und im Nachhinein reflektiert noch eine weit größere Menge an Korrespondenz die Bedeutung des stattgehabten Ereignisses. Noch am Abreisetag des 31. Januar dankt Rilke Katharina Kippenberg, der Dame des Hauses, für die erfolgte Gastfreundschaft; es ist dies der erste in einer Serie von 139 Briefen, die Rilke spezifisch an die Frau seines Verlegers richten wird. ${ }^{22}$ Und an Kippenberg selbst vermeldet Rilke von seiner Folgedestination Berlin sogleich, das „schöne herzliche Haus“ des Verlegers erscheine ihm nun bereits als „eine recht feste Stelle [...] in allem Beweglichen, Ungewissen“. „Sie glauben nicht, wie sicher und ruhig mich die Erfahrung Ihrer Gastfreundschaft macht, die ich mir viel vergegenwärtige mit aller Erinnerung an weit vorausreichendes, fruchtbares Einverstehen." ${ }^{23}$ Auch der Antwortbrief des Verlegers bekräftigt die durch gemeinsame Leipziger Tage geschlossene freundschaftliche Bindung als Manifestation einer bereits zuvor gefassten wechselseitigen Neigung: „Das herzliche Einvernehmen, dessen Nachgefühl in meiner Frau und mir so warm lebendig ist, war wohl vorbereitet, von beiden Seiten: durch herzliche Verehrung für den Dichter von unserer, durch Vertrauen von Ihrer Seite.“24

Und doch ist, bei all diesen epistolaren Bekundungen von Herzlichkeit, aus der persönlichen Nähe zwischen Verleger und Autor nicht gerade eine wirkliche Freundschaft erwachsen; dafür klingt das beteuerte Einvernehmen zu stereotyp und zu förmlich, auch wenn Kippenberg für diesmal dies Schreiben sogar eigenhändig und handschriftlich ausfertigte, statt wie sonst meistens seine Briefe an Rilke als geschäftliche Korrespondenz zu diktieren. Wie sehr emotionale Anteile auch hineinwirken mögen, so verbleibt die Beziehung beider gleichwohl im arbeitstechnischen Rahmen einer vertraglich definierten Zusammenarbeit, die durch

22 Rilke u. Kippenberg 1954.

23 Rilke an Kippenberg, 1. Februar 1910; BW Kippenberg, Bd. I, 186.

24 Kippenberg an Rilke, 8. Februar 1910; BW Kippenberg, Bd. I, 188. 
Rilkes episodischen Gastaufenthalt im Turmzimmer bald eine Art von mythischem Gütesiegel erhält.

Wiederholt gerät Rilke ins Schwärmen, wenn in seinen weiteren Briefen „,von dem festen Versammeltsein der leipziger Tage“ die Rede ist, ${ }^{25}$ als ließe sich mit der Erinnerung auch die ideale Arbeitsruhe der damaligen Gastwochen aufs Neue evozieren. Und auch der Verleger seinerseits knüpft mehrfach in emphatischer Weise an das Erlebnis des Dichtergastes an, darf er sich selbst und seinem Hause nun doch sogar eine gewisse Mitwirkung bei der Fertigstellung des Malte Laurids Brigge zugutehalten:

Mein lieber Herr Rilke, [/] Ein eigenhändiges Wort des Dankes, daß Sie so lange bei uns und der unsrige waren. Die sechs Wochen Ihres Hierseins, geistige herzliche Wochen, die der noch nicht völlig Materie gewordene Malte Laurids doppelt verschönte, werden uns für alle Zeit unvergeßlich sein. ,Es ist vortheilhaft, den Genius zu bewirten ${ }^{\text {- }}$ auch in unserem Hause lebt nun etwas von Ihnen, das ihm bleiben wird. ${ }^{26}$

Wie stark auch ein nüchtern kalkulierender Geschäftsmensch wie Kippenberg jenen Leipziger Aufenthalt seines bedeutendsten Autors nachträglich geradezu ins Mythische überhöht, wird etwa schon an einem Indiz wie der grotesk überdehnten angegebenen Zeitdauer ablesbar, die tatsächlich keineswegs sechs, sondern gerade einmal zweieinhalb Wochen umfasste, wovon überdies noch die Tage eines Vortrags in Jena abzuziehen waren. ${ }^{27}$ Es sind derlei briefliche Diskursmanöver, die der kurzen Episode des persönlichen Zusammenseins die Bedeutung eines magisch-symbolischen Kontrakts verleihen. Ihr Verhältnis geht über die geschäftliche Beziehung nun deutlich hinaus, wenn auch nicht gerade ins Freundschaftliche; es ist, als könne der Verleger sich durch die temporäre Beherbergung des Dichters in einer entscheidenden Arbeitsphase eine mitzeugende (oder zumindest mit auf die Welt bringende) Rolle beim Werkprozess zuschreiben.

Diese maieutische Funktion des Verlegers kann dieser vor allem für den ersten Leipziger Gastaufenthalt Rilkes im Januar 1910 geltend machen; der Sommeraufenthalt im Folgejahr, als Rilke die letzten August- und ersten Septembertage wiederum das „Turmzimmer“ der Verlegervilla in der Richterstraße bezieht, ist längst nicht von gleicher Bedeutungstiefe, da ihm kein entsprechender werkbildender Schaffensmoment innewohnt, im Gegensatz zu der vorangegangenen Arbeit am Brigge-Manuskript. Dass sich der Verleger auf die damalige Mitwirkung am dichterischen Schaffen einiges zugutehielt, wird durch einige weitere Briefpassagen

25 Rilke an Kippenberg, 14. Februar 1910; BW Kippenberg, Bd. I, 190.

26 Kippenberg an Rilke, 20. März 1910; BW Kippenberg, Bd. I, 198.

27 Schnack 2009, 340. 
belegt, in welchen Kippenberg später wiederholt und stets emphatisch auf Rilkes ersten Turmzimmer-Aufenthalt zu sprechen kommt. So bittet er den Autor bei einem Widmungsexemplar für seine Ehefrau Katharina Kippenberg, nach Möglichkeit ausdrücklich Bezug zu nehmen „auf die Vollendung des Malte Laurids im historischen Turmzimmer und auf das erste Vorlesen des vollendeten Werkes in der Richterstraße 2." 28 Rilke seinerseits nimmt die stehende Formulierung von den damaligen „Turmzimmer“-Tagen mehrfach auf, um das produktive Band mit seinem Verleger zusehends enger zu knüpfen und sich für gelegentliche Wiederholungen des Gastaufenthaltes bereits in Vormerkung zu bringen. Insbesondere nach der Rückkehr von Schloss Duino sondiert Rilke die Zugverbindungen Paris-Leipzig und „bezöge“ gerne „für ein paar Tage die Thurmwohnung“, wie er den Verleger wissen lässt; ${ }^{29}$ ein Plan, welchem er noch weitere briefliche Absichtserklärungen folgen lässt, ehe es im Juli 1914 nochmals zu einem Besuch in Leipzig kommt, am epochalen Vorabend jener Kriegsjahre, die den Bruch Rilkes mit Deutschland einleiten werden, seine Zeiten des Reisens und Leidens und seine schließliche Neu-Ansiedlung in der Schweiz.

Auch in das Verhältnis zu Kippenberg sollten die Weltkriegsjahre und die aggressive nationalistische Haltung des Deutschen Reiches, mit welcher der Verleger kräftig sympathisierte, die scharfe Zäsur eines nicht mehr gänzlich zu kittenden Dissenses bringen. Rilke verstand sich, gerade unter den Bedrängnissen der Kriegsjahre und ihrer Folgen, dezidiert als ein pazifistischer Zeitgenosse von europäischer Kultur und Gesinnung. Dass der Dichter hierin mit Kippenberg nicht d'accord ging, bekundete er zwar in Briefäußerungen, die an ideologiekritischer Deutlichkeit nichts zu wünschen übrig lassen, doch war er zugleich gegenüber dem Verleger stets bemüht, dieser Divergenz in ihrer beider Verhältnis keinen allzu großen Raum zu geben, um nicht die Fortdauer der so produktiven Zusammenarbeit und des literarischen Einverständnisses zu gefährden.

Über seine Tätigkeit im Münchner Kriegsarchiv, in das Rilke im Januar 1916 abkommandiert worden war, hatte er Kippenberg trotz der damit bewerkstelligten Front-Verschonung nur Negatives zu berichten - allzu vehement drängten sich in jener Institution die Zwänge propagandistischer Beschönigung hervor.

Dort ist nun meine Lage (Büreaustunden von 9 bis 3) äußerlich bequemer und besser, aber wahrscheinlich unhaltbar, wenn es mir nicht gelingt zu ganz mechanischen Abschreibe- oder Registrier-Arbeiten versetzt zu werden; denn der Dicht-Dienst, zu dem sich die Herren seit

28 Kippenberg an Rilke, 10. April 1913; BW Kippenberg, Bd. I, 394.

29 Wie „[...] gerne käme ich mit diesem französischen Zug und bezöge für ein paar Tage die Thurmwohnung: denn wievieles wäre da fürs Gespräch der Abende“ (Rilke an Kippenberg, 10. März 1913; BW Kippenberg, Bd. I, 384). 
anderthalb Jahren geübt haben, ist mir völlig unmöglich. Ich mag ihn nicht beschreiben, er ist sehr dürftiger und zweideutiger Natur, und eine Abstellung alles Geistigen (wie das in der Kaserne der Fall war) scheint beneidenswerth neben diesem schiefen und unverantwortlichen Mißbrauch schriftlicher Bethätigung. Die Herren selbst nennen es ,das Heldenfrisieren', lange graute ihnen, nun haben sie sich dazu überwunden und werfens aus dem Handgelenk. ${ }^{30}$

Einer derartigen Korrumpierung seiner literarischen Existenz mochte sich Rilke trotz der äußerlich zunehmend bedrängenden Situation nicht unterwerfen. Nicht nur in ideologischer Hinsicht, sondern ebenso in den alltäglichen Aspekten des kulturellen Lebens waren im Deutschland der Kriegsjahre Deformationen zu verzeichnen, die auch in den ersten Friedensjahren und beim Aufbau der politisch weiterhin umstrittenen Republik noch nachwirkten. Insofern war es nicht ohne innere Folgerichtigkeit, wenn Rilke sich in den Nachkriegsjahren um einen Neubeginn in der kulturell vielfältigen Schweiz bemühte. Die darin sich ausdrückende Distanz gegenüber Deutschland war wiederum für Kippenberg ein steter Stein des Anstoßes, so dass er die gelegentlich geäußerten Rückkehrpläne energisch aufgriff und den Dichter darin zu bekräftigen suchte, letztlich allerdings erfolglos, wie man weiß. ${ }^{31}$ Mit gewisser Resignation, aber auch generöser Milde akzeptiert der Verleger vier Jahre später, dass im Hinblick auf zeitpolitische Fragen keine Übereinkunft mehr zu erzielen war. „Lassen Sie es uns nicht dauern, daß wir in politischen Dingen uns nicht oder noch nicht verstehen. In unseres Vaters Hause sind viele Wohnungen, und da wir in der des Herzens so einträchtig zusammen hausen, so mag uns das mehr als genug sein. “32

\section{5}

Man wird die Symbolik der Ortswahl bei Rilkes später Niederlassung im Turm zu Muzot über dem Rhonetal wohl nicht überbewerten, wenn man darin - aus markanter Ferne evoziert - auch einen elegisch mitschwingenden Nachklang des

30 Rilke an Kippenberg, 15. Februar 1916; BW Kippenberg, Bd. II, 46.

31 Im Herbst 1920 begrüßt Kippenberg freudig Rilkes „Absicht baldiger Rückkehr“ und fordert ihn auf, „den Weg über Leipzig zu nehmen“. Es lockt die Anknüpfung an ehemalige Turmtage: „Das rote Zimmer oben und das Turmzimmer erwarten sie sehnsüchtig. Ich kann mir denken, dass es Ihnen nicht leicht fällt, jetzt nach Deutschland zurückzukehren, und die Schweiz, die Ihnen so besonders gastlich gewesen ist, zu verlassen, aber ich habe das bestimmte Gefühl, dass es richtig so ist. Wie sehr wünschte ich mir, Ihnen, wenn Sie hier sind, nicht nur in äusserlichen Dingen raten, sondern Sie auch im Innern bestärken zu können.“ (1. September 1920; BW Kippenberg, Bd. II, 173).

32 Kippenberg an Rilke, 2. Juni 1924; BW Kippenberg, Bd. II, 341. 
Leipziger Turmzimmers erkennt. Rilke hat die topographische Auszeichnung erhaben und ,ausgesetzt“ liegender Orte und Gebäude stets hochgeschätzt und als poetisch inspirierend betrachtet, weil sie ein zwar räumlich abgeschottetes, aber zugleich ganz auf Empfang gerichtetes Dasein, gewissermaßen ein Existieren im ursprünglichen Wortsinne ermöglichte. Zu den für ihn lebenslang weiterwirkenden Konsequenzen des ersten Leipziger Aufenthaltes gehörte zum einen die nahezu exklusive Bindung an seinen wichtigsten Verleger, der sich über viele Jahre hinweg und letztlich erfolgreich bemüht hatte, auch Rilkes anderweitig vergebene Publikationen sukzessive in sein eigenes Haus zu holen. Zum zweiten hatte sich aus der Erfahrung der produktiven Gastzeit bei Kippenberg für Rilke offenkundig eine Art Arbeitsmodell entwickelt, das auf die temporäre Beherbergung durch mäzenatische Freunde zur Förderung einer örtlich markanten, poetisch inspirierenden Situation hinauslief.

Schon als Rilke anfangs 1912 bei seinem längeren Aufenthalt auf Schloss Duino als Gast der Gräfin Thurn und Taxis in rauschhaften Schüben die Konzeption und Niederschrift der ersten Elegien begann, wiederholte sich dieses Modell eines hospitalen Mäzenatentums, nun aber aufgrund der Zuwendung einer anderen Gönnerin. Der Verleger wird sogleich hellhörig und möchte die entstandenen Gedichte baldmöglichst in einer geeigneten Weise publiziert sehen, doch Rilke wehrt dies vehement ab, da sich der Komplex dessen, was später die Duineser Elegien genannt würde, für ihn noch in statu nascendi befindet. Der Dichter schreibt seinem Verleger im Mai 1912 eine gleichermaßen dessen Neugier noch anfachende wie sie vertröstende Mitteilung, die trotz des zwischen beiden eigentlich erreichten Vertrauensverhältnisses wieder auf den aus Rilkes Korrespondenzen vertrauten Mechanismus des Wechselspiels von Annäherung und Distanzierung zurückfällt.

Die ,Elegie', lieber Freund, ich freu mich, daß Sie sie in Wien kennen gelernt haben; es ist noch eine zweite da, Kassner bekam sie in Duino vorgelesen, und die Fürstin Taxis und er hatten die stärkste Überzeugung auch für diese zweite, gaben ihr vielleicht sogar den Vorzug vor der früheren. Für mich sind diese Arbeiten, was seiner Zeit das Stundenbuch war in seinem Entstehen, ein wirklicher Anhalt; der erste Theil des ,Stundenbuchs' hat Jahre und Jahre in Freundeshänden gelegen, ehe er mit den späteren Abschnitten zu dem Buch zusammenund herauskam, ähnlich möcht ich es mit den Elegien halten, schön im Geheimen, ich fühle, es soll so sein, und Sie werden mich, weiß ich, gewähren lassen und mirs nicht zur Laune anschreiben. ${ }^{33}$

Das Manöver des Dichters gegenüber seinem Verleger ist mehrschichtig; der Anreiz des Interesses für das neue Werk wird gesteigert einerseits durch die Erwähnung konkurrierender Kenner wie Rudolf Kassner oder der Gräfin Taxis, zum anderen

33 Rilke an Kippenberg, 13. Mai 1912; BW Kippenberg, Bd. I, 340. 
durch die gleichzeitig und im Widerspruch dazu fortgesetzte Attitüde der Geheimhaltung. Hierbei verfolgt Rilke die Strategie, in der Zusammenarbeit mit Kippenberg und trotz der ökonomischen Abhängigkeit von diesem gleichwohl eine maximale autorschaftliche Souveränität für sich selbst zu sichern. Der Dichter behält sich allein die Entscheidung darüber vor, ob, wann und wie ein entstehendes Werk in die Öffentlichkeit entlassen werden solle; er verweist auf den Entstehungsvorgang des Stundenbuchs, um darzulegen, dass ihm ein dem allgemeinen Publikum vorgeschalteter Freundeskreis von gewissermaßen ,vorkostenden' Primäradressaten als ein unentbehrlicher Bestandteil des poetischen Reifungsprozesses gilt.

Neben dieser produktionsästhetischen Abschirmung eines noch in der weiteren Ausarbeitung befindlichen Werkkomplexes stand im Falle der Elegien einer frühzeitigen Publikation auch der Umstand entgegen, dass Rilke die schon vorliegenden Gedichte als organische Bestandteile eines noch zu bildenden Ensembles betrachtete, das er erst mit dem Wiedergewinnen des damaligen Schaffensaufschwungs würde fertigstellen können. Deshalb erneuert er bei fortgesetzten, ungeduldigen Anfragen des Verlegers zu wiederholten Malen seine restriktive Haltung gegenüber dem Thema eines Vorabdrucks aus dem Elegien-Bestand. „Seien Sie nicht enttäuscht,“ schreibt der Dichter im Sommer 1917, „wenn ich Ihnen keine Elegie für den Almanach schicke. Diese Gedichte müssen mein innerer Besitz bleiben dürfen, bis einmal der ganze Zusammenhang herausgestellt sein wird und wir ihn in Einem zur Erscheinung bringen." 34

Die jahrelange Abschottung eines noch andauernden Werkprozesses gegenüber dem auf Publikation dringenden Literaturvermittler war eine spannungsvolle, psychologisch durchaus heikle Angelegenheit, zumal Rilke seinen Verleger zum Verwalter seiner gesamten Honorarangelegenheiten bestellt hatte und deshalb in finanzieller Hinsicht so vollkommen von ihm abhängig war, dass er für alle größeren Ausgaben zunächst das Placet seines Vertrauten einholen musste. Auch in dieser Hinsicht zeigt sich Rilke als betont naiver, unfertiger Marktteilnehmer und kokettiert in hilfloser Selbstironie mit seiner Unbedarftheit in allen finanziellen Angelegenheiten, wenn er dem Verleger im Zusammenhang des Wunsches einer größeren Zahlung mit keinesfalls übertriebener Selbstbezichtigung mitteilt: „Sie sehen, auf was für einer niederen unmündigen Stufe meine kapitalistischen Vorstellungen sich abspielen.“ “35

Der Verleger rückte in den publizistischen und ökonomischen Betätigungen Rilkes zunehmend in die Rolle eines väterlichen Vertrauten und sogar Vormunds, der für den Dichter nicht nur das gesamte Management und die professionelle

34 Rilke an Kippenberg, 5. Juli 1917; BW Kippenberg, Bd. II, 71.

35 Rilke an Kippenberg, 2. November 1915; BW Kippenberg, Bd. II, 38. 
Außendarstellung übernahm, sondern auch bezüglich erzielbarer Einnahmen und zu tätigender Ausgaben immer wieder strenge Ratschläge erteilte, die sein Autor zwar aufgrund ihrer Kompetenz wertschätzte, gleichwohl aber oft genug mit der Geste infantiler Eigenwilligkeit in den Wind schlug. Auch dieses Rollenspiel war geeignet, ein tatsächliches Bewusstsein für die Mechanismen und Möglichkeiten des literarischen ,Marktes‘ bei Rilke gar nicht aufkommen zu lassen. Er nutzte die geschäftliche Versiertheit Kippenbergs vielmehr, um sich in maximalem Kontrast zu diesem als in wirtschaftlichen Fragen vollständig unbedarft zu positionieren, eine Haltung, die sich in dieser langjährigen brieflichen Partnerschaft durch deren intrinsische Differenzdynamik sicherlich noch verstärkt haben dürfte.

Stets blickte der Autor mit Bangen auf die materiellen Grundlagen seiner Existenz; er leistete sich den größtmöglichen Abstand zu dem Erfordernis, die Ergebnisse der eigenen Arbeit sogleich öffentlich taxiert, in Einnahmen, Umsätze und Gewinnmargen umgerechnet sehen zu müssen. Zugleich aber konnte der Schriftsteller eine gewisse Faszination für die verwertungstechnische Seite des Publikationsgeschäftes nicht verhehlen; vor allem dann, als sich abzeichnete, dass seine Lyrik in den 1910er Jahren ein beständig wachsendes Publikum fand und entsprechend gestiegene Absatzzahlen erwirtschaftete, mehrten sich auch die interessierten Rückfragen an den Verleger.

Doch waren und blieben Rilkes Vorstellungen seiner dichterischen Existenz auch in den publizistisch erfolgreicheren Jahren produktionsästhetisch letztlich an ein mäzenatisches Modell der Gönnerschaft geknüpft und rezeptionsseitig auf einen kleinen, ausgewählten Adressatenkreis bezogen. Obwohl er die eigene dichterische Produktion als eruptive, auf rare Inspirationsmomente gegründete und schubweise vonstatten gehende Schöpfungsvorgänge stilisierte, kam dem freundschaftlich-kollegialen Umfeld bei der ,Erst-Verkostung` eigener Werke eine wichtige Testfunktion zu. Für diesen Schwellenbereich zur Öffentlichkeit schuf sich Rilke hauptsächlich durch Briefe, erst in zweiter Linie durch persönliche Begegnungen ein zwar überschaubares, aber hoch effizientes Netzwerk von literarischen Gesprächspartnerinnen und -partnern.

Diese Beziehungen, innerhalb derer auch die Korrespondenz zu seinem Verleger Kippenberg funktional anzusiedeln ist, ermöglichten dem Autor eine Art präpublizistischen Inkubationsraum, in dem er Gedankengänge, Motivmuster, Textproben und ganze Werkreihen in zwanglosen Experimentalkonstellationen ausarbeiten und erproben konnte. Neben den poetologisch konsultativen Korrespondenzen - und natürlich den im engeren Sinne libidinös-intimen Kontakten erwiesen sich hierbei in werkbildender Hinsicht vor allem diejenigen Kommunikationsstränge von besonderer Schubkraft, welche mit der Organisation von Hospitalität verbunden waren und auf konkrete Schreiborte referierten: das Turmzimmer von Leipzig, Schloss Duino an der Adria, schließlich das Walliser Turmgemach. Das 
Wiederaufleben eines mäzenatischen Modells konnte dabei aus gesellschaftlichen Gründen nicht mehr unter feudal-aristokratischen Vorzeichen stehen, wie sehr Rilke auch im freundschaftlichen Verkehr noch auf die Insignien von Nobilität zu setzen tendierte. Es waren letztlich gerade diese widerstrebenden Züge an seiner Modernität, die Rilke eine dichterische Arbeit ermöglichten, welche sich durchaus in zeitgenössischen, modernen Produktions- und Vertriebsstrukturen bewegte, wie die beachtlichen wirtschaftlichen Erfolge belegen, die der Insel-Verleger mit den Neuen Gedichten und mit dem Cornet, aber auch mit den im heimischen Turmzimmer vom Dichtergast vollendeten Aufzeichnungen des Malte Laurids Brigge erzielte.

\section{Literatur}

Bohnenkamp, Anne u. Waltraud Wiethölter (Hg.) (2008). Der Brief-Ereignis \& Objekt. Katalog der Ausstellung im Freien Deutschen Hochstift - Frankfurter Goethe-Museum 2008. Frankfurt a. M. Honold, Alexander u. Irmgard Wirtz (Hg.) (2019). Rilkes Korrespondenzen. Göttingen.

Kammer, Stephan (2019). „Schlechte Einheit. Zur Epistolarität des Erzählens in Rilkes Aufzeichnungen des Malte Laurids Brigge“, in: Rilkes Korrespondenzen. Hg. v. Alexander Honold u. Irmgard Wirtz. Göttingen: 33-52.

Keller, Gottfried (1996). Sämtliche Werke. Hg. v. Peter Villwock. Bd. 3: Der grüne Heinrich. Zweite Fassung. Frankfurt a. M.

Martus, Steffen (2007). Werkpolitik. Zur Literaturgeschichte kritischer Kommunikation von 17. bis ins 20. Jahrhundert mit Studien zu Klopstock, Tieck, Goethe und George. Berlin.

Rilke, Rainer Maria u. Katharina Kippenberg (1954). Briefwechsel. Hg. v. Bettina von Bornhard. Wiesbaden.

Rilke, Rainer Maria (1995). Briefwechsel mit Anton Kippenberg. Hg. v. Ingeborg Schnack u. Renate Scharffenberg. 2 Bde. Frankfurt a. M. u. Leipzig.

Schnack, Ingeborg (2009). Rainer Maria Rilke. Chronik seines Lebens und seines Werkes 1875-1926. Erweiterte Neuausgabe von Renate Scharffenberg. Frankfurt a. M. u. Leipzig.

Schuster, Jörg (2014). „Kunstleben“. Zur Kulturpoetik des Briefs um 1900 - Korrespondenzen Hugo von Hofmannsthals und Rainer Maria Rilkes. Paderborn.

Strobel, Jochen (Hg.) (2006). Vom Verkehr mit Dichtern und Gespenstern. Figuren der Autorschaft in der Briefkultur. Heidelberg.

Vellusig, Robert (2000). Schriftliche Gespräche. Briefkultur im 18. Jahrhundert. Wien. 